\title{
Disrupted Attention to Other's Eyes is Linked to Symptoms of ADHD in Childhood
}

\author{
Matilda A. Frick ${ }^{1}$ D $\cdot$ Karin C. Brocki ${ }^{1} \cdot$ Linda Halldner Henriksson ${ }^{2,3} \cdot$ Johan Lundin Kleberg $^{4,5}$
}

Accepted: 7 January 2022

(c) The Author(s) 2022

\begin{abstract}
Attention-deficit/hyperactivity disorder (ADHD) is associated with impaired social interaction. Other's eyes are important for understanding the social world. Here, we examined concurrent and longitudinal links between attention to other's eyes and symptoms of ADHD and comorbid externalizing and internalizing symptoms. Eighty-two 8 to 13-year-old children (40\% with ADHD) participated. The latency to a first gaze shift to and away from the eye region of human faces, when primed to look at either the eyes or the mouth, was recorded with eye tracking. Parents rated ADHD, externalizing and internalizing symptoms at the time of testing and at 2-year follow-up. The results show that longer looking at the eyes before reorienting was specifically associated with concurrent and future symptoms of inattention, even when accounting for comorbid symptoms. We conclude that the temporal microstructure of attention to other's eyes is altered in children with symptoms of ADHD, which may contribute to social impairments.
\end{abstract}

Keywords Attention-deficit/hyperactivity disorder (ADHD) · Eye tracking · Face perception · Anxiety · Internalizing · Externalizing symptoms

\section{Introduction}

Attention-deficit/hyperactivity disorder (ADHD) is associated with impairments in multiple life domains, including school and occupational performance, peer relations and increased risk for delinquency such as alcohol and substance abuse [1-3]. Although not part of the diagnostic criteria, social interaction impairments are common in ADHD across the life span [4]. For example, children with ADHD are often excluded from peer groups because of difficulties

Matilda A. Frick

matilda.frick@psyk.uu.se

1 Division of Emotion Psychology, Department of Psychology, Uppsala University, Box 1225, 75142 Uppsala, Sweden

2 Department of Clinical Science, Child and Adolescent Psychiatry, Umeå University, Umeå, Sweden

3 Department of Medical Epidemiology and Biostatistics, Karolinska Institutet, Stockholm, Sweden

4 Department of Clinical Neuroscience, Centre for Psychiatry Research, Karolinska Institutet, \& Stockholm Health Care Services, Region Stockholm, Stockholm, Sweden

5 Department of Molecular Medicine and Surgery, Karolinska Institutet, Stockholm, Sweden with turn-taking, behavioral regulation during play [5, 6] and regulation of both positive and negative affect [7]. For humans, faces are one of the most important sources of learning about the social environment [8]. Disrupted attention to faces could therefore be an underlying mechanism of the social interaction impairments observed in ADHD. A small literature has examined how children with ADHD attend to faces. For example, previous studies have reported that children with ADHD respond to faces with altered gaze behavior [9], autonomic [10] and brain activity [11, 12]. Recently, Gui and colleagues [13] reported that 14-monthold toddlers who had elevated symptoms of ADHD at a follow-up looked longer at images of faces among distractors before reorienting, suggesting difficulties with disengagement of attention. These studies do not indicate that faceperception atypicalities in ADHD depend on the emotional expression of the face [e.g. 9, 10].

The eye region is the most informative part of the face [e.g. 14]. Recent research suggests that children with ADHD may show specific impairments related to processing of other's eye gaze. For example, children with ADHD often fail to attend to others' eyes during emotion recognition [15] and are not using others' gaze direction to guide their attention [16]. Consequently, intervention programs teach parents to 
increase periods of sustained eye contact with their children with ADHD as a means of increasing compliance [17].

Information from the eye region is believed to be a major source of learning and experience-dependent development of the social brain [14] and could therefore contribute to the developmental course of ADHD. However, to our knowledge, no longitudinal studies have so far examined visual attention to eyes as a longitudinal predictor of ADHD symptoms. Altered face perception may be a common phenotypic characteristic of ADHD and autism spectrum disorder (ASD) [18], as reduced attention to faces in the presence of competing non-social information, reduced eye contact, and a diminished ability to understand information expressed in the eye region is commonly seen in ASD [19].

Visual scanning of faces follows a relatively uniform sequence. Initially, human eyes trigger quick and reflexive gaze shifts towards the eyes, a process called social orienting [14]. Subsequently, gaze is reoriented from the eyes to other aspects of the face (typically the mouth) or to the focus of the other person's gaze. Disruptions to both these processes (orienting and reorienting) could potentially interfere with social perception and interaction [20]. It is therefore important not only to examine whether ADHD and associated symptom dimensions are linked to atypical attention to eyes, but also to establish at what stage. That is, how early in the attentional sequence potential deviations can be observed and whether orienting towards the eyes, away from the eyes or both is affected. Further, this pattern could vary as a function of the depicted emotion in the face. For instance, theories of social attention in children with anxiety disorders have focused on three processes - vigilance, avoidance, and delayed disengagement [21,22]. Vigilance would be manifested as rapid gaze shifts towards potentially threatening stimuli such as angry faces, avoidance as a tendency to orient away from the same stimuli, and delayed disengagement as increased dwell time before reorienting, perhaps reflecting problems with shifting attention from potential threats. These theories have mainly been tested in studies comparing the relative allocation of attention between whole faces with a threatening expression and various non-social control stimuli $[22,23]$, and not in relation to eye gaze specifically. A recent study of adolescents with social anxiety disorder [20] found that this group differed from healthy controls by taking longer time to reorient from eyes, supporting the delayed disengagement hypothesis. It is not known whether a similar pattern of attention pertains also to other forms of anxiety symptoms, such as generalized anxiety, when comorbid with ADHD.

ADHD is highly overlapping with externalizing symptoms of oppositional defiant disorder (ODD) and conduct disorder (CD) and internalizing symptoms such as anxiety. ODD refers to a pattern of anger, irritability and defiance towards caregivers and other adults with a typical onset in early childhood [24]. CD refers to a more severe pattern of antisocial behavior, characterized by lack of respect for others, reduced empathy and rule breaking [24]. Different anxiety disorders are usually highly correlated and not always easily distinguishable, suggesting that they are closely linked to a common internalizing factor [25]. Generalized anxiety is characterized by excessive and widespread worrying [24]. Symptoms of generalized anxiety commonly debut in childhood and are closely linked to trait neuroticism, which represents a broad risk factor for internalizing disorders [26], making it a suitable construct for the current study. These co-occurring externalizing and internalizing symptom dimensions are both associated with atypical eye gaze processing [20,27]. It is therefore not clear whether the link between ADHD symptoms and atypical attention to others' eyes is due to core ADHD difficulties or to comorbid externalizing and internalizing symptoms. However, scarce research of facial emotion recognition have suggested that the challenges children with ADHD face within this area may be better explained by comorbid externalizing symptoms than by ADHD symptoms per se [e.g. 15].

There is some evidence that symptoms of externalizing disorders are related to a reduced tendency to orient to eyes during free-viewing tasks, and that this in turn is related to impaired emotion recognition [e.g. 15]. For example, Dadds et al. [27] reported that boys with callous unemotional traits, a severe form of externalizing symptoms characterized by low emotionality, poor affective empathy, and proactive aggression, were less likely to orient to others' eyes, which in turn affected their emotion recognition. Other studies have suggested that personality traits associated with enhanced reward sensitivity and interpersonal aggression are linked to prolonged periods of eye contact once it is established [28]. Given the relationships between externalizing symptoms, enhanced reward sensitivity, and interpersonal aggression, externalizing symptoms may be connected to delayed orienting away from eyes, although it is not clear from the previous literature whether this effect would be modulated by the emotional expression of the face.

The developmental correlates of altered eye gaze in ADHD remains largely unexamined. Human eyes are arguably one of the most important sources of social learning of the environment [14]. Consequently, maladaptive attention to other's eyes during childhood could potentially interfere with social learning, thereby exacerbating core ADHD and comorbid symptom levels over time and as such contribute to non-optimal development. On the other hand, flexible attention to others' eyes could potentially increase opportunities for symptom reduction through adaptive social learning. In both scenarios, attention to others' eyes would be expected to predict subsequent change in ADHD, externalizing, and internalizing symptoms but in different directions. There is currently a lack of studies examining this matter. 
Even though ADHD, ODD/CD, and generalized anxiety refer to diagnostic categories, it has been argued that empirically psychopathological constructs are best conceptualized as dimensional traits [29]. As such, obtaining a dimensional perspective adds both statistical power and a more fine-grained understanding of connections between symptoms dimensions that are interrelated yet separable by different developmental trajectories and associated risks [30, 31].

To sum up, previous studies have linked symptoms of ADHD and comorbid symptoms of ODD/CD and anxiety to atypical eye contact, but many questions remain. For instance, the specificity of these atypicalities to different symptom dimensions; the sequency stage at which they occur; the direction of the effects (enhanced vs. diminished eye contact); and the extent to which atypical eye contact predict change in symptom levels across development. We used an eye preference task (EPT) in which a group of school-aged children (aged 8-13 years), oversampled for ADHD-diagnoses ( $40 \%$ had a previous diagnosis of ADHD) were primed to look towards the eyes or the mouth of depicted faces expressing different emotions (i.e. angry, happy, and neutral). Eye preference was operationalized as the latency to orient away (OA) from the eyes when the initial point of gaze was in the eyes region and the latency to orient towards (OT) the eyes when the initial point of gaze was in the mouth region. We set out to examine the orienting measures globally (i.e. a composite of all expressed emotions) and separately for each expressed emotion.

We examined the following research questions:

1. Is eye preference concurrently and specifically (i.e., with control for the other symptom domains) related to symptoms of ADHD, ODD/CD, and generalized anxiety?

2. Is eye preference longitudinally and specifically related to symptoms of $\mathrm{ADHD}, \mathrm{ODD} / \mathrm{CD}$, and generalized anxiety at follow-up two years later (T2)?

3. Is eye preference related to the change in symptoms of ADHD, ODD/CD, and generalized anxiety between $\mathrm{T} 1$ and T2 (with and without control for the other symptom domains at T1)?

As for the direction of effects, we expected symptoms of ODD/CD to be linked to delayed orienting away from the eyes when the eye region was primed $(\mathrm{OA})$ and delayed orienting towards eyes when the mouth was primed (OT). Further, we expected symptoms of generalized anxiety to be related to delayed orienting away from eyes when the eye region was primed (OA). No directed hypotheses were specified for the other research questions or for specific emotions due to inconsistencies in the previous literature.

\section{Method}

\section{Participants}

An initial sample of 84 children aged 8 to 13 years old participated in the study at T1. Due to technical errors with the EPT paradigm, the final sample consisted of the 82 individuals $\left(\mathrm{M}^{\mathrm{age}}=10.49\right.$ years, $\mathrm{SD}=1.36 ; 59$ boys, $72 \%$ ) who were successfully assessed with the EPT. Thirty-four children (41\%) had a diagnosis of ADHD (24 boys, $71 \%$ ). At T2, which took place 2 years later, 67 children remained in the study (retention rate $82 \%$; 46 boys, $69 \% ; \mathrm{M}^{\mathrm{age}}=12.57$ years, $\left.\mathrm{SD}=1.42\right)$, of which $27(40 \%)$ had a diagnosis of ADHD (18 boys, 67\%). The remaining children had slightly higher socio-economic status (SES) compared to the dropouts $(4.23$ vs. $3.52, \mathrm{t}(79)=2.46$, $p=0.016$ ), but did not differ on any of the other study variables $(p s>0.16)$. The children with ADHD were recruited through ads in the local newspaper $(n=10)$, on Facebook $(n=2)$, through interest groups $(n=4)$, and through childpsychiatry and pediatric outpatient clinics $(n=18)$. Diagnostic status was confirmed through medical records in 15 cases, for the remaining 19 cases the parents reported that their children had been formally assessed in regular child psychiatric or pediatric care and had received an ADHD diagnosis. The children without a diagnosis were recruited through the population registry and information letters about the study were sent to 1,000 families in the local area. Of 116 interested responders, 50 children without psychiatric disorders, matched with the ADHD group for age, sex, and SES were enrolled in the study.

Of the children with ADHD, $80 \%$ were on regular medication for ADHD (methylphenidate, atomoxetine, dexamphetamine, or extended-release guanfacine). Individuals with stimulant medication were asked to refrain from medication on the day of assessment, yet 10 (29\%) were medicated during the EPT. These visits were still completed as the test leader or the parent did not deem it feasible to postpone them until after a washout phase. Analyses showed that medication on the day of testing was unrelated to all EPT measures and was therefore left out of further analyses. Four individuals reported a comorbid disorder of autism spectrum disorder. Removing these did not change the results in a profound way and they were therefore kept in the final sample. As for other comorbid disorders, three participants reported an anxiety disorder, one a sleep disorder, and one a language disability. The regional ethics board (EPN 2014/285, Uppsala, Sweden) approved the study. All legal guardians gave written informed consent, and all children gave oral informed consent to participation. 


\section{Procedure}

The study consisted of a 2-h visit to the Department of Psychology, Uppsala University or to Kista BUMM, a pediatric outpatient clinic in Kista, Stockholm. The children were assessed with the EPT and various other tasks presented elsewhere $[10,32,33]$. Parents (mothers $[n=62,75.6 \%]$, fathers $[\mathrm{n}=2,2.4 \%]$, or both parents together $[\mathrm{n}=19$, $23.2 \%]$ ) completed questionnaire data on symptom levels at $\mathrm{T} 1$ and $\mathrm{T} 2$. For participation, the families received gift certificates worth $\sim 20$ USD for each time point.

\section{Measures}

\section{Experimental Paradigm: The Eye-Preference Task}

The experimental paradigm was adapted from a previous study [20]. Stimulus images were human faces cropped to show only the inner region of the face. Each trial started with a fixation cross presented for one second before the onset of the stimulus image (see Fig. 1). The stimulus image was then presented, positioned in relation to the fixation cross so that the participant was looking either in the eye region ( $50 \%$ of trials) or in the mouth region (50\% of trials). Stimulus images were shown for $2 \mathrm{~s}$. Participants completed 30 trials in each condition (OA and OT), mixed together in a randomized order, interleaved with stimuli from other tasks not analyzed here, which did not include faces. The stimulus images were equally balanced between three emotional expressions: angry, happy, and neutral. The children were instructed to just watch what happened on the screen. The eye-tracking session lasted approximately five minutes.

Recording and Analysis of Eye-Tracking Data Eye-tracking data were recorded at a sample rate of $60 \mathrm{HZ}$ using a corneal-reflection eye tracker (Tobii TX120, Tobii, Danderyd, Sweden). Saccades were identified using a custom I-VT filter with velocity threshold set to $30 \%$ s. Saccadic latency was defined as the time of onset of the first saccade away from the initial cued point of gaze. Two variables were extracted: 1) OA, the latency to orient away from the eyes (and to the mouth) when the initial point of gaze was cued to the eyes, and 2) OT, the latency to initiate/orient a saccade towards the eyes when the initial point of gaze was cued to the mouth. Trials were discarded if participants were not looking at the fixation cross at the onset of the facial image, or if at least $25 \%$ valid fixation time was registered at either the primed or the non-primed region. Cut-off was set to 10 valid trials of OA and OT respectively for inclusion in analyzes (discarding five participants on OA and four on OT). Invalid trials could result from movement artefacts, excessive blinks or equipment failure. Setting the cut-off at 8,10 , or 12 did not affect the results significantly.

After exclusion, two complementary eye tracking metrics can be calculated: the latency to initiate a saccade to the nonprimed region and the proportion of trials in which a saccade to that region is initiated (as opposed to trials during which gaze is maintained at the primed region). Preliminary analyses in the present sample showed strong overlap between these variables in the OA $(r=-0.95)$ and OT $(r=-0.91)$ both $p<0.0001$, in that individuals who were quicker to orient to the non-primed region were also more likely to do so, rather than to maintain their gaze at the primed region. Due to the substantial overlap between these variables, only the latency measures were included in the current study. Cronbach's alpha was $\alpha=0.93$ for OA and $\alpha=0.67$ for OT, the former considered excellent and the latter in the lower range for what may be considered acceptable.

\section{Symptom Measures at $\mathrm{T} 1$ and $\mathrm{T} 2$}

ADHD Symptoms We used the ADHD Rating Scale-5 for Children and Adolescents [34] to assess ADHD symptoms at T1 and T2. Parents rated the 18 symptoms listed in the DSM-5 [24] on a scale from 0 to 3 . Nine items concern inattention and 9 concern hyperactivity/impulsivity. We used the mean of each symptom domain, which were analyzed
Fig. 1 Stimuli and overview of the experiment. All trials started with a fixation cross presented for $1 \mathrm{~s}$ followed by the stimulus image presented for $2 \mathrm{~s}$. Stimulus images were positioned relative to the fixation cross so that participants initial point of gaze was either within the eye region (A, Orient-away condition, $\mathrm{OA})$ or in the mouth region (B, Orient-towards condition, OT). Dependent variables were the latency to orient away from eyes (OA) or towards the eyes (OT)

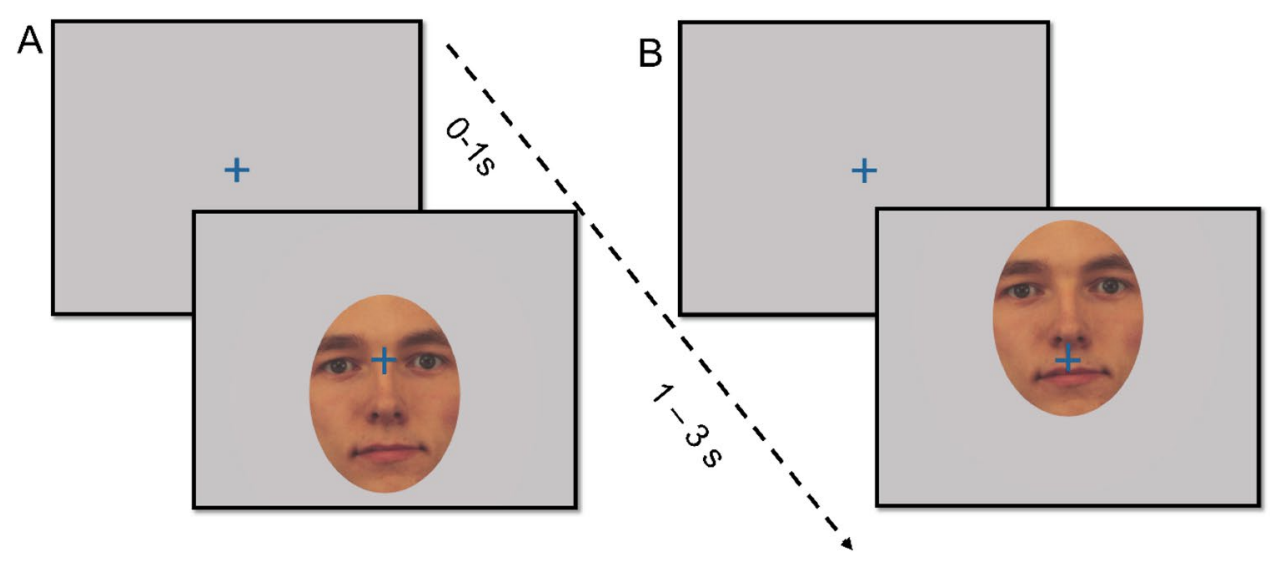


separately due to its different developmental trajectories [30]. Cronbach's alpha was $\alpha=0.94$ to 0.96 .

Externalizing Symptoms We used the Swanson, Nolan, and Pelham scale-IV (SNAP-IV) ODD subscale [35] to assess ODD symptoms. Six age-appropriate DSM-5 symptoms of $\mathrm{CD}$ was added to the scale. Parents rated the eight ODD symptoms and six CD symptoms on a scale from 0 to 3 . To obtain a broad measure of externalizing behaviors we used the mean across the two domains, which were significantly correlated $(r h o=0.55, p<0.0001$ at $\mathrm{T} 1$ and $r h o=0.38$, $p=0.001$ at T2). Cronbach's alpha was $\alpha=0.88$ at both time points.

Generalized Anxiety We used the generalized-anxiety subscale (comprising 6 items) from Spence Children's Anxiety Scale (SCAS) [36] to assess anxiety in the children. Parents rated anxiety levels in their children on a scale from 0 to 3 . Cronbach's alpha was $\alpha=0.77$ at $\mathrm{T} 1$ and $\alpha=0.86$ at $\mathrm{T} 2$.

Change Scores Change scores were calculated for each symptom domain by subtracting scores at $\mathrm{T} 1$ from $\mathrm{T} 2$. As such, values $>0$ reflect an increase of symptoms across time and values $<0$ reflect a symptom decrease.

\section{Control Variables}

Sex, age, intelligence quotient (IQ) and SES were included as control variables in the models if significantly correlated with any of the main study variables.

Intelligence Quotient We used Block design and Information from WISC-IV [37] as a proxy for IQ. These subtests had the highest loading on non-verbal and verbal IQ in the Swedish validation of WISC-IV [37]. The scaled scores (mean $=10 ; \mathrm{SD}=3$ ) for the two tests were significantly correlated $(r=0.35, p=0.002)$ and collapsed into one measure for IQ.

Socio-Economic Status Parents rated their own and the child's other parent's highest level of education $(1=$ elementary school, $2=$ vocational high school, $3=$ theoretical high school, $4=$ post high-school studies [not University], and $5=$ college/University $)$ and yearly income $(1=>10,000$ USD; $2=10,000$ to 20,000 USD; $3=20,000$ to 30,000 USD, $4=30,000$ to 40,000 USD, $5=40,000$ to 50,000 , and $6=>50,000$ USD). The mean across both parents was used as a proxy for SES.

\section{Analytic Strategy}

The analysis plan was pre-registered in the Open Science Framework (https://osf.io/tg8mw/). Analyzes were performed in IBM SPSS 26 (IBM, Corp., Armonk, NY, USA). $Z$ score \pm 3 were considered outliers and as such winsorized, that is replaced with the highest/lowest values not considered outliers [38]. Skewness and kurtosis were examined using guidelines by Field [39]. Residuals were plotted and visually inspected for deviant patterns to ensure that linear regressions could be used. Cronbach's alpha was used to examine internal consistency of the scales and eye tracking variables. Due to a relatively small sample size, the possibility of imputing missing values was scrutinized to increase power. We present descriptive statistics based on original data. All further analyzes are carried out on imputed data. Group differences based on independent samples $t$ tests are presented for descriptive purposes. To increase power and based on a dimensional perspective on ADHD, all main analyses are dimensional and carried out on the full sample. We used correlations to examine bivariate associations and hierarchical regression analyses to examine independent contributions. To reduce the number of predictors in the models, only predictors and covariates with significant correlations to a predictor or criterion variable were included in the models. We report unstandardized $b$ s as output.

\section{Results}

\section{Preliminary Results}

Ten outliers (representing eight different measures from eight different participants) were found and winsorized. Most measures had excessive skewness, kurtosis, or both. Hence, Spearman correlations were used. Visual inspection of residual plots revealed no deviant patterns and therefore parametric regressions could be used. Nine percent of the data were missing. Little's MCAR test was non-significant $(p=0.071)$, indicating that data was missing completely at random and five sets of imputations were made using standard fully conditional specification (FCS-Standard) [40], in which all study variables were used in the imputation phase. See Table 1 for descriptive statistics and Table 2 for correlations. OA regarding all emotions were significantly correlated ( $r h o s=0.30$ to $0.58, p \mathrm{~s}<0.05$ ) and collapsed to one measure for OA. OT regarding all emotions were also significantly correlated ( $r h o=0.42$ to $0.61, p s<0.05$ ) and collapsed to one measure for OT. As most of these correlations were in the moderate range, unity and diversity of orienting as a function of emotion seem to be present. As such, analyses were conducted with both total scores of OA and OT as well as with scores for the respective emotions. As shown in Table 1, mean symptom levels decreased slightly from $\mathrm{T} 1$ to $\mathrm{T} 2$ for all symptom domains at the group level. The largest decrease was a reduction of hyperactivity/impulsivity for 
Table 1 Descriptive statistics

\begin{tabular}{|c|c|c|c|c|c|c|c|c|c|c|}
\hline & \multicolumn{3}{|c|}{ Total sample } & \multicolumn{3}{|c|}{ ADHD group } & \multicolumn{3}{|c|}{ Control group } & \multirow{2}{*}{$\begin{array}{l}\text { Group difference } \\
p\end{array}$} \\
\hline & $n$ & $M$ & $S D$ & $n$ & $M$ & $S D$ & $n$ & $M$ & $S D$ & \\
\hline SES & 81 & 4.11 & 1.01 & 33 & 3.84 & 1.02 & 48 & 4.29 & 0.98 & .051 \\
\hline IQ & 78 & 9.66 & 2.57 & 32 & 9.52 & 3.25 & 46 & 9.76 & 2.01 & .681 \\
\hline OA Anger & 77 & 768 & 287 & 33 & 807 & 311 & 44 & 705 & 264 & .106 \\
\hline OA Нарру & 81 & 762 & 324 & 34 & 789 & 311 & 47 & 744 & 336 & .537 \\
\hline OA Neutral & 71 & 804 & 346 & 26 & 884 & 354 & 45 & 762 & 337 & .170 \\
\hline OA & 81 & 769 & 241 & 34 & 803 & 236 & 47 & 746 & 244 & .279 \\
\hline OT Anger & 81 & 436 & 223 & 34 & 476 & 267 & 47 & 407 & 183 & .197 \\
\hline OT Happy & 79 & 491 & 244 & 31 & 525 & 277 & 48 & 453 & 218 & .095 \\
\hline OT Neutral & 82 & 394 & 181 & 34 & 395 & 196 & 48 & 393 & 171 & .967 \\
\hline OT & 82 & 442 & 187 & 34 & 476 & 209 & 48 & 417 & 168 & .159 \\
\hline T1 Inatt & 81 & 0.99 & 0.81 & 33 & 1.78 & 0.60 & 48 & 0.44 & 0.33 & $<.001$ \\
\hline T1 Hyp/Imp & 81 & 0.86 & 0.83 & 33 & 1.67 & 0.67 & 48 & 0.29 & 0.28 & $<.001$ \\
\hline T1 ODD/CD & 81 & 0.44 & 0.41 & 33 & 0.72 & 0.47 & 48 & 0.26 & 0.22 & $<.001$ \\
\hline T1 GAD & 79 & 1.66 & 0.42 & 33 & 1.79 & 0.47 & 46 & 1.57 & 0.36 & .020 \\
\hline T2 Inatt & 67 & 0.94 & 0.75 & 27 & 1.58 & 0.65 & 40 & 0.45 & 0.37 & $<.001$ \\
\hline T2 Hyp/Imp & 67 & 0.67 & 0.72 & 27 & 1.25 & 0.68 & 40 & 0.28 & 0.41 & $<.001$ \\
\hline $\mathrm{T} 2 \mathrm{ODD} / \mathrm{CD}$ & 67 & 0.38 & 0.37 & 27 & 0.55 & 0.44 & 40 & 0.23 & 0.25 & .011 \\
\hline T2 GAD & 67 & 1.62 & 0.52 & 27 & 1.80 & 0.61 & 40 & 1.52 & 0.41 & .031 \\
\hline Inatt CS & 67 & -0.04 & 0.43 & 27 & -0.15 & 0.55 & 40 & 0.04 & 0.31 & .112 \\
\hline Hyp/Imp CS & 67 & -0.17 & 0.43 & 27 & -0.45 & 0.50 & 40 & -0.02 & 0.28 & $<.001$ \\
\hline ODD/CD CS & 67 & -0.06 & 0.26 & 27 & -0.11 & 0.33 & 40 & -0.02 & 0.20 & .197 \\
\hline GAD CS & 65 & -0.05 & 0.35 & 27 & 0.03 & 0.37 & 38 & -0.08 & 0.34 & .235 \\
\hline
\end{tabular}

SES socio-economic status, $O A$ orienting away from eyes, $O T$ orienting towards eyes, Inatt inattention, Hyp/Imp hyperactivity/impulsivity, $O D D / C D$ oppositional defiant disorder/conduct disorder, $C S$ change score (across T1 and T2). OA and OT are measured in milliseconds; all other measures are based on mean values from the rating scales

the ADHD group, which was significantly larger than the reduction for the typically developing children.

At group level, participants oriented slower from the eyes $(\mathrm{OA})$ of neutral than happy $(\mathrm{t}(76)=6.82, p<0.001)$ and angry faces $(\mathrm{t}(76)=4.40, p<0.001)$, whereas no difference was found between happy and angry faces $(\mathrm{t}(76)=0.95$, $p=0.340$ ). When primed to look at the mouth (OT), participants were slower to orient to the eyes of happy than angry $(\mathrm{t}(76)=3.24, p=0.002)$ and neutral $(\mathrm{t}(76)=2.92, p=0.005)$ faces. No difference was found between angry and neutral faces $(\mathrm{t}(76)=0.34, p=0.736)$.

\section{Concurrent Associations Between Eye Preference and Symptoms}

OA was significantly correlated with inattention at $\mathrm{T} 1$ $(r h o=0.28, p=0.018)$, in that a longer latency to orient away from the eyes (when primed to look at the eyes) was associated with more symptoms. This was the case for OA anger $(r h o=0.34, p=0.003)$ and OA happy $(r h o=0.26$, $p=0.024)$. Further, OA anger was significantly correlated with hyperactivity/impulsivity ( $r h o=0.30, p=0.010$ ). OT was not significantly related to concurrent symptom levels.

To examine specific relations between eye preference and symptoms, we performed a hierarchical regression model with OA as criterion variable and age, SES, hyperactivity/impulsivity and ODD/CD as covariates entered in the first step and inattention as the main predictor entered in the final step. This was informed by the pattern of significant correlations, where inattention was the only predictor with a significant correlation with OA. The results show that inattention was independently associated with OA (see Table 3). As OA anger and OA happy were both significantly correlated with inattention, we ran an exploratory analysis to examine independent effects of emotion in that we regressed inattention on OA anger and OA happy. The results showed that OA anger $(b=0.001$, $p=0.018, \mathrm{R}^{2} \Delta=0.083$ ) was independently associated with inattention at $\mathrm{T} 1$ whereas happy was not $(p=0.693)$. 


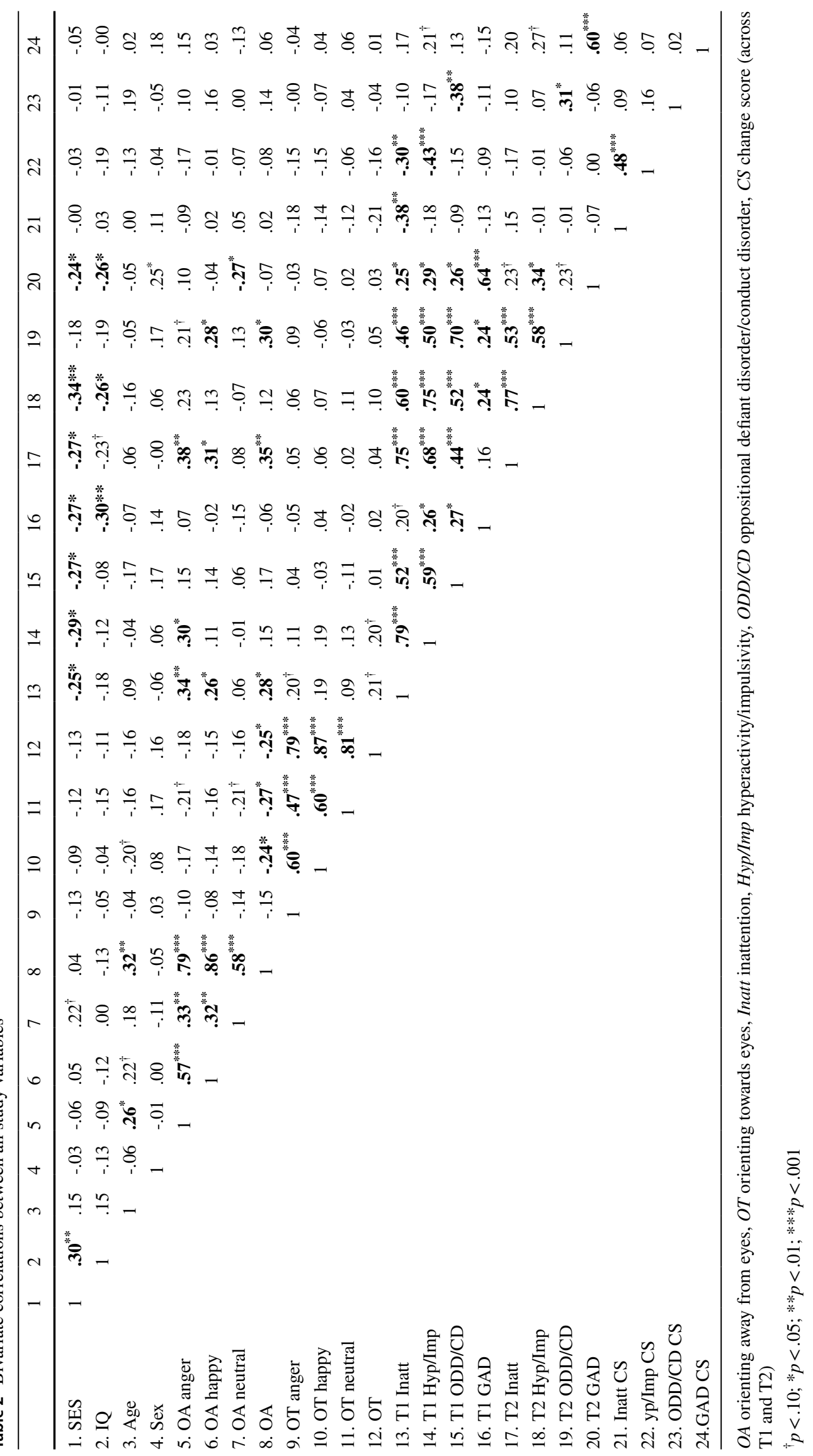


Table 3 The final steps of hierarchical regression models with latency to orient away from eyes $(\mathrm{OA})$ as criterion variable

\begin{tabular}{|c|c|c|c|c|c|c|c|c|}
\hline & \multicolumn{4}{|c|}{ Predictors at $\mathrm{T} 1$} & \multicolumn{4}{|c|}{ Predictors at $\mathrm{T} 2$} \\
\hline & $b$ & $p$ & Adjusted $\mathrm{R}^{2}$ & $\mathrm{R}^{2} \Delta$ & $b$ & $p$ & Adjusted $\mathrm{R}^{2}$ & $\mathrm{R}^{2} \Delta$ \\
\hline Step 2 & & & 0.154 & & & & 0.098 & \\
\hline Constant & 105.39 & .645 & & & 171.97 & .543 & & \\
\hline Age & 37.71 & .059 & & & 45.09 & .037 & & \\
\hline SES & 42.74 & .107 & & & 46.69 & .109 & & \\
\hline Hyp/Imp & -77.05 & .228 & & & -88.13 & .277 & & \\
\hline GAD & & & & & -26.86 & .649 & & \\
\hline $\mathrm{ODD} / \mathrm{CD}$ & 83.34 & .327 & & & 79.10 & .372 & & \\
\hline Inattention & 136.04 & .037 & & .050 & 129.32 & .049 & & .049 \\
\hline
\end{tabular}

SES socio-economic status, Hyp/Imp hyperactivity/impulsivity, GAD symptoms of generalized anxiety disorder, $O D D / C D$ symptoms of oppositional defiant disorder/conduct disorder. Significant results in bold

\section{Longitudinal Associations Between Eye Preference at T1 and Symptoms at T2}

OA was significantly correlated with inattention $(r h o=0.35, p=0.005)$ and $\mathrm{ODD} / \mathrm{CD}(r h o=0.30$, $p=0.017)$ at $\mathrm{T} 2$, in that a longer latency to orient away from the eyes was associated with more symptoms two years later. As for specific emotions, inattention at T2 was significantly correlated with OA anger $(r h o=0.38$, $p=0.005)$ and OA happy ( $r h o=0.31, p=0.016)$ and OA happy was significantly correlated with ODD/CD at T2 ( $r h o=0.28, p=0.018)$. Further, OA neutral was significantly correlated with generalized anxiety $(r h o=-0.27$, $p=0.045$ ), in that a shorter latency to look away from neutral faces was associated with more symptoms. OT was not significantly related to symptom levels at $\mathrm{T} 2$.

To examine specific relations between eye preference and symptoms at $\mathrm{T} 2$, we performed a hierarchical regression model with $\mathrm{OA}$ as criterion variable, and age, SES, hyperactivity/impulsivity, and GAD as covariates entered in the first step and inattention and ODD/CD as the main predictors entered in the final step. The results show that inattention was independently associated with OA whereas ODD/CD was not (see Table 3). Exploratory analyses regarding specific emotions showed that when included in the same step in a hierarchical regression, neither OA anger nor OA happy made independent contributions $(p s<0.130)$ to inattention at $\mathrm{T} 2$, indicating a common rather than specific effects of emotion.

OA and OT were unrelated to change in symptoms across $\mathrm{T} 1$ and $\mathrm{T} 2$ (see Table 2). As attention to eyes was unrelated to change in symptoms we did not perform additional analyzes. We conclude that this finding indicates that altered attention to eyes does not exacerbate symptoms over time.

\section{Discussion}

In the current study, we set out to examine concurrent and longitudinal relations between attention to other's eyes and core symptoms of ADHD and comorbid externalizing $(\mathrm{ODD} / \mathrm{CD})$ and internalizing (generalized anxiety) symptoms. Our main results indicate that a longer latency to orient away from the eyes, when primed to look at the eye region was associated with higher levels of inattentive symptoms. This was found both concurrently and at a two-year follow up, and remained with control for the other symptom domains. This association was present for emotional (angry and happy) but not neutral faces and seems primarily driven by a tendency to 'get stuck' in the eye region of angry faces, which was also related to concurrent high levels of hyperactivity/impulsivity. The latency to orient towards the eyes when primed to look at the mouth was unrelated to symptom levels, indicating that our finding is not a sign of a general reduced orienting speed. Attention to the eyes was not associated with exacerbation of symptoms across time.

Together, these results demonstrate that ADHD symptoms in childhood are linked to alterations in the temporal microstructure of social attention. Adaptive face processing requires a quick and coordinated sequence of gaze shifts between facial regions and objects in the environment, and disruptions to any stage of this process could potentially lead to social interaction impairments. Interestingly, whereas the latency to orient away from eyes was specifically linked to symptoms of inattention both concurrently and longitudinally, no links were found to the latency to seek eye contact. These two stages in the sequence of social attention have different neurodevelopmental correlates. Rapid orienting to eyes is observed already in infancy and is believed to be driven by a largely subcortical network of brain regions sensitive to the coarse visual characteristics of eyes [14, 41, 42]. 
Disruptions at this stage have been linked to autism [43-45] (but see [46]) and Williams syndrome, a genetic disorder with early emerging social alterations [47]. In contrast, the latency to reorient from eyes is believed to be modulated by higher order cognitive processes including spatial attention and motivation. Delayed reorienting from eyes in children with higher levels of ADHD symptoms therefore seemingly indicates disruption to this normative process. A similar pattern of attention was recently reported in a study of social anxiety disorder (SAD) in children aged 10-17 years using the same paradigm as the current study [20]. Here, children with SAD were slower than healthy controls to orient from the eye region during the earliest time stages of attention but did not differ in the latency to orient to eyes. As such, different alterations of face processing may be present for ADHD and ASD, whereas ADHD and SAD may overlap on a phenotypical level. Importantly, symptoms of SAD and inattention are overlapping, and may share similar aetiological factors [48]. It should be noted that, at the group level, children in the present study were quicker to reorient from the eyes when viewing emotional than neutral faces.

A longer latency to orient away from the eyes was consistently and independently associated with inattentive symptoms, concurrently and longitudinally, which indicates a robust relation. Further, this association was most pronounced for angry faces. These results are aligned with attenuated tonic arousal as a potential mechanism underlying our result. Several previous studies have reported both behavioral and physiological evidence for hypoarousal [33, 49-51], and hypoarousal has been suggested to underlie enhanced attention to other's eyes in other developmental disorders [52]. A second potential mechanism explaining this result, is impaired disengagement of attention from stimuli eliciting emotional arousal. In support of this interpretation, previous studies reported similar effects in youth with rumination, a population which like ADHD is associated with reduced cognitive control [53], and recently also in social anxiety disorder [20]. Of note, delayed orienting away from angry faces was also concurrently (but not longitudinally) related to hyperactivity/impulsivity. The lack of a prospective relation to hyperactivity/impulsivity may be explained by a commonly observed developmental decline in this symptom dimension [30].

In contrast to our hypotheses, we did not find a specific connection between attention to eyes and comorbid externalizing or internalizing symptoms. As our sample was oversampled for ADHD diagnoses rather than high levels of comorbid symptoms, we may not have had sufficient power to detect such an effect due to a restricted range and variation for these measures. There were however prospective associations between longer orienting away from eyes and symptoms of ODD/CD at T2, suggesting a link between the two. This link seems to be shared or overlapping with ADHD symptoms at $\mathrm{T} 2$, proposing a general rather than specific association between these constructs. In sum, our results propose a specific association to inattention rather than to comorbid symptoms, which may be secondary to symptoms of ADHD, but not to attention to other's eyes.

The lack of an exacerbating effect suggests that deviant eye gaze does not contribute to escalation of symptoms over time. This may infer that eye gaze on this time scale is not involved in pro-social learning (hence no connection to change in ODD/CD) or emotion processing (hence no connection to generalized anxiety). Rather, our results may suggest that altered eye gaze in ADHD could be a trait-like factor that persists over time but does not affect symptom levels over time.

An interesting question for future studies is whether altered eye gaze processing in ADHD can be modulated by stimulant- and non-stimulant medication and other treatments. Previous studies have shown that both types of treatment can reduce core symptoms of ADHD, as well as associated comorbidity [54]. However, individual variability exists and effects on social skills and peer interaction difficulties are not sufficiently studied. It is not known whether proximal changes in social attention can mediate more distant treatment effects. If such relations would be found, social attention metrics could be feasible as biomarkers in treatment research. It should be noted that, in addition to its links to ADHD symptoms, the OA measure in the present study showed excellent internal psychometric consistency (Cronbach's $\alpha=0.93$ ), suggesting that it may be feasible as a marker task in treatment research. The OT measure, however, had lower internal consistency (Cronbach's $\alpha=0.67$ ), which calls for further examination before it can be proposed as a reliable measure in future studies. The role of social attention metrics in diagnostic assessments should be scrutinized, as subtyping individuals with or without these impairments could guide tailored treatments. Futures studies should also directly compare children with ADHD and autism, since previous studies have linked the latter condition to atypical eye contact [43].

As for limitations, we wish to acknowledge that symptom ratings from multiple informants (such as teachers, parents and the affected children themselves) could have given a more complete picture than parent-ratings alone. Relatedly, self-ratings of internalizing could potentially have provided better sensitivity. A broader assessment of internalizing symptoms would have been informative, as that would have enabled a distinction between social and non-social aspects of anxiety. In addition, the relatively low variation in comorbid symptoms is a limitation and we do not have information about which interventions (medical, psychological or pedagogical) our subjects have received between $\mathrm{T} 1$ and $\mathrm{T} 2$. Earlier and repeated assessment of eye-preference could further inform on the effect of gaze on pro-social learning and 
emotion processing. Finally, we did not correct for multiple testing, based on the increased risk of making type- 2 errors with such a modest sample. Instead, we believe that our preregistration of analyses reduces the risk of providing falsepositive results.

\section{Summary}

The current study explored the foundation of impaired social interactions in ADHD. We examined associations between attention to other's eyes and symptoms of core ADHD symptoms and comorbid externalizing and internalizing symptoms in a sample of 82 eight to 13-year-olds oversampled for ADHD. The latency to a first gaze shift to and away from the eye region, when primed to looked at the eyes or the mouth, was recorded with eye tracking. The main results show that longer looking at the eye region before reorienting was independently associated with concurrent and longitudinal symptoms of inattention with control for the other symptom domains, and significantly correlated with inattention and externalizing symptoms two years later. The results propose that the temporal microstructure of attention to other's eyes is altered in children with symptoms of ADHD, which in turn may contribute to social impairments.

Acknowledgements We wish to thank Mozaffar Hessami, MD and Kista BUMM for help with participant recruitment and master students Matilda Knutar Lewander, Moa Sjöborg, Elin Hansson, and Anna Larnebring for help with recruitment and data collection.

Funding Open access funding provided by Uppsala University. This research was supported by Grant 2017/29 from Jerringfonden to Karin C. Brocki.

\section{Declarations}

Conflict of interest The authors have no conflicts of interest to declare that are relevant to the content of this article.

Informed Consent Informed consent was obtained from all individual participants included in the study.

Ethical Approval All procedures performed in the study involving human participants were in accordance with the ethical standards of the national research committee (The regional ethics board [EPN 2014/285, Uppsala, Sweden] approved the study) and with the 1964 Helsinki declaration and its later amendments.

Open Access This article is licensed under a Creative Commons Attribution 4.0 International License, which permits use, sharing, adaptation, distribution and reproduction in any medium or format, as long as you give appropriate credit to the original author(s) and the source, provide a link to the Creative Commons licence, and indicate if changes were made. The images or other third party material in this article are included in the article's Creative Commons licence, unless indicated otherwise in a credit line to the material. If material is not included in the article's Creative Commons licence and your intended use is not permitted by statutory regulation or exceeds the permitted use, you will need to obtain permission directly from the copyright holder. To view a copy of this licence, visit http://creativecommons.org/licenses/by/4.0/.

\section{References}

1. Bernardi S, Faraone SV, Cortese S, Kerridge BT, Pallanti S, Wang S, Blanco C (2012) The lifetime impact of attention-deficit hyperactivity disorder: results from the National Epidemiologic Survey on Alcohol and Related Conditions. Psychol Med 42(4):875-887. https://doi.org/10.1017/S003329171100153X

2. Fletcher JM (2014) The effects of childhood adhd on adult labor market outcomes. Health Econ 23(2):159-181. https://doi.org/10. 1002/hec. 2907

3. Sundquist J, Ohlsson H, Sundquist K, Kendler KS (2015) Attention-deficit/hyperactivity disorder and risk for drug use disorder: a population-based follow-up and co-relative study. Psychol Med 45(5):977-983. https://doi.org/10.1017/S0033291714001986

4. Hoza B (2007) Peer functioning in children with ADHD. J Pediatr Psychol 32(6):655-663. https://doi.org/10.1093/jpepsy/jsm024

5. Diamantopoulou S, Henricsson L, Rydell A-M (2005) ADHD symptoms and peer relations of children in a community sample: examining associated problems, self-perceptions, and gender differences. Int J Behav Dev 29(5):388-398. https://doi.org/10.1177/ 01650250500172756

6. Gardner DM, Gerdes AC (2015) A review of peer relationships and friendships in youth with ADHD. J Atten Disord 19(10):844855. https://doi.org/10.1177/1087054713501552

7. Cole PM, Zahn-Waxler C, Smith KD (1994) Expressive control during a disappointment: variations related to preschoolers' behavior problems. Dev Psychol 30(6):835-846. https://doi.org/ 10.1037/0012-1649.30.6.835

8. Olsson A, Knapska E, Lindström B (2020) The neural and computational systems of social learning. Nat Rev Neurosci 21(4):197212. https://doi.org/10.1038/s41583-020-0276-4

9. Muszkat M, de Mello CB, de Muñoz POL, Lucci TK, David VF, de Siqueira JO, Otta E (2015) Face Scanning in autism spectrum disorder and attention deficit/hyperactivity disorder: human versus dog face scanning. Front Psychiatry. https://doi.org/10.3389/fpsyt. 2015.00150

10. Kleberg JL, Frick MA, Brocki KC (2020) Increased pupil dilation to happy faces in children with hyperactive/impulsive symptoms of ADHD. Dev Psychopathol. https://doi.org/10.1017/S0954 579420000036

11. Ichikawa H, Nakato E, Kanazawa S, Shimamura K, Sakuta Y, Sakuta R, Yamaguchi MK, Kakigi R (2014) Hemodynamic response of children with attention-deficit and hyperactive disorder (ADHD) to emotional facial expressions. Neuropsychologia 63:51-58. https://doi.org/10.1016/j.neuropsychologia.2014.08. 010

12. Tye C, Mercure E, Ashwood KL, Azadi B, Asherson P, Johnson MH, Bolton P, McLoughlin G (2013) Neurophysiological responses to faces and gaze direction differentiate children with ASD, ADHD and ASD + ADHD. Dev Cogn Neurosci 5:71-85. https://doi.org/10.1016/j.den.2013.01.001

13. Gui A, Mason L, Gliga T, Hendry A, Ali JB, Pasco G, Shephard E, Curtis C, Charman T, Johnson MH, Meaburn E, Jones EJH (2020) Look duration at the face as a developmental endophenotype: elucidating pathways to autism and ADHD. Dev Psychopathol 32(4):1303-1322. https://doi.org/10.1017/S0954579420000930 
14. Senju A, Johnson MH (2009) The eye contact effect: mechanisms and development. Trends Cogn Sci 13(3):127-134. https://doi.org/ 10.1016/j.tics.2008.11.009

15. Airdrie JN, Langley K, Thapar A, van Goozen SHM (2018) Facial emotion recognition and eye gaze in attention-deficit/hyperactivity disorder with and without comorbid conduct disorder. J Am Acad Child Adolesc Psychiatry 57(8):561-570. https://doi.org/10. 1016/j.jaac.2018.04.016

16. Marotta A, Pasini A, Menotti E, Pasquini A, Pitzianti MB, Casagrande $M$ (2017) Controlling attention to gaze and arrows in attention deficit hyperactivity disorder. Psychiatry Res 251:148-154. https://doi.org/10.1016/j.psychres.2017.01.094

17. Kapalka GM (2004) Longer eye contact improves ADHD children's compliance with parents' commands. j Atten Disord 8(1):17-23. https://doi.org/10.1177/108705470400800103

18. Ioannou C, Seernani D, Stefanou ME, Riedel A, van Tebartz Elst L, Smyrnis N, Fleischhaker C, Biscaldi-Schaefer M, Boccignone G, Klein C (2020) Comorbidity matters: social visual attention in a comparative study of autism spectrum disorder, attention-deficit/ hyperactivity disorder and their comorbidity. Front Psychiatry 11:545567. https://doi.org/10.3389/fpsyt.2020.545567

19. Chita-Tegmark M (2016) Social attention in ASD: a review and meta-analysis of eye-tracking studies. Res Dev Disabil 48:79-93. https://doi.org/10.1016/j.ridd.2015.10.011

20. Kleberg JL, Högström J, Sundström K, Frick A, Serlachius E (2021) Delayed gaze shifts away from others' eyes in children and adolescents with social anxiety disorder. J Affect Disord 278:280-287. https://doi.org/10.1016/j.jad.2020.09.022

21. Högström J, Nordh M, Lindal ML, Taylor E, Serlachius E, Kleberg JL (2019) Visual attention to emotional faces in adolescents with social anxiety disorder receiving cognitive behavioral therapy. PLoS ONE 14(11):e0225603. https://doi.org/10.1371/journal. pone. 0225603

22. Lau JYF, Waters AM (2017) Annual research review: an expanded account of information-processing mechanisms in risk for child and adolescent anxiety and depression. J Child Psychol Psychiatry 58(4):387-407. https://doi.org/10.1111/jcpp.12653

23. Armstrong T, Olatunji BO (2012) Eye tracking of attention in the affective disorders: a meta-analytic review and synthesis. Clin Psychol Rev 32(8):704-723. https://doi.org/10.1016/j.cpr.2012. 09.004

24. American Psychiatric Association (2013) Diagnostic and statistical manual of mental disorder, 5th edn. American Psychiatric Publishing, Washington, DC

25. Griffith JW, Zinbarg RE, Craske MG, Mineka S, Rose RD, Waters AM, Sutton JM (2010) Neuroticism as a common dimension in the internalizing disorders. Psychol Med 40(7):1125-1136. https://doi.org/10.1017/S0033291709991449

26. Hale WW, Klimstra TA, Meeus WHJ (2010) Is the generalized anxiety disorder symptom of worry just another form of neuroticism?: A 5-year longitudinal study of adolescents from the general population. J Clin Psychiatry 71(07):942-948. https://doi.org/10. 4088/JCP.09m05506blu

27. Dadds MR, Allen JL, McGregor K, Woolgar M, Viding E, Scott $S$ (2014) Callous-unemotional traits in children and mechanisms of impaired eye contact during expressions of love: a treatment target? J Child Psychol Psychiatry 55(7):771-780. https://doi.org/ 10.1111/jcpp. 12155

28. Terburg D, Hooiveld N, Aarts H, Kenemans JL, van Honk J (2011) Eye tracking unconscious face-to-face confrontations: dominance motives prolong gaze to masked angry faces. Psychol Sci 22(3):314-319. https://doi.org/10.1177/0956797611398492

29. Krueger RF, Piasecki TM (2002) Toward a dimensional and psychometrically-informed approach to conceptualizing psychopathology. Behav Res Ther 40(5):485-499. https://doi. org/10.1016/S0005-7967(02)00016-5

30. Brocki KC, Forslund T, Frick M, Bohlin G (2019) Do individual differences in early affective and cognitive self-regulation predict developmental change in ADHD symptoms from preschool to adolescence? J Atten Disord 23(13):1656-1666. https://doi.org/ $10.1177 / 1087054717693372$

31. Leve LD, Kim HK, Pears KC (2005) Childhood temperament and family environment as predictors of internalizing and externalizing trajectories from ages 5 to 17. J Abnorm Child Psychol 33(5):505-520. https://doi.org/10.1007/s10802-005-6734-7

32. Frick MA, Brocki KC (2019) A multi-factorial perspective on ADHD and ODD in school-aged children: what is the role of cognitive regulation, temperament, and parental support? J Clin Exp Neuropsychol 41(9):933-945. https://doi.org/10.1080/13803 395.2019.1641185

33. Kleberg JL, Frick MA, Can Brocki K. C (2020) Auditory warning signals normalize eye movements in children with ADHD? Eur Child Adolesc Psychiatry. https://doi.org/10.1007/ s00787-020-01484-w

34. DuPaul GJ, Power TJ, Anastopoulos AD, Reid R (2016) ADHD Rating Scale-5 for Children and Adolescents: Checklists, Norms, and Clinical Interpretation; Guilford Publications

35. Bussing R, Fernandez M, Harwood M, Hou W, Garvan CW, Eyberg SM, Swanson JM (2008) Parent and teacher SNAP-IV ratings of attention deficit hyperactivity disorder symptoms: psychometric properties and normative ratings from a school district sample. Assessment 15(3):317-328. https://doi.org/10.1177/ 1073191107313888

36. Spence SH (1998) A Measure of Anxiety Symptoms among Children. Behav Res Ther 36(5):545-566. https://doi.org/10.1016/ S0005-7967(98)00034-5

37. Wechsler D, Wechsler D (2007) Wechsler Intelligence Scale for Children Fourth Edition. The Swedish Version, vol 2007. Pearson, Stockholm

38. Tabachnick BG, Fidell LS (2001) Using multivariate analysis. Harper Collins College Publishers, California State University Northridge, Northridge

39. Field A (2013) Discovering statistics using IBM SPSS statistics. SAGE, London

40. Huque MH, Carlin JB, Simpson JA, Lee KJ (2018) A comparison of multiple imputation methods for missing data in longitudinal studies. BMC Med Res Methodol 18(1):168. https://doi.org/10. 1186/s12874-018-0615-6

41. Itier RJ, Batty M (2009) Neural bases of eye and gaze processing: the core of social cognition. Neurosci Biobehav Rev 33(6):843863. https://doi.org/10.1016/j.neubiorev.2009.02.004

42. Tomalski P (2015) Developmental trajectory of audiovisual speech integration in early infancy. A review of studies using the McGurk Paradigm. Psychol Lang Commun 19(2):77-100. https:// doi.org/10.1515/plc-2015-0006

43. Guillon Q, Hadjikhani N, Baduel S, Rogé B (2014) Visual social attention in autism spectrum disorder: insights from eye tracking studies. Neurosci Biobehav Rev 42:279-297. https://doi.org/10. 1016/j.neubiorev.2014.03.013

44. Hedger N, Dubey I, Chakrabarti B (2020) Social orienting and social seeking behaviors in ASD. A meta analytic investigation. Neurosci Biobehav Rev 119:376-395. https://doi.org/10.1016/j. neubiorev.2020.10.003

45. Jónsdottir LK, Neufeld J, Falck-Ytter T, Kleberg JL (In Press) Autistic children quickly orient away from oth eyes and mouths during face observation. J Autism Dev Disord

46. Johnson MH (2014) Autism: Demise of the Innate Social Orienting Hypothesis. Curr Biol 24(1):R30-R31. https://doi.org/10. 1016/j.cub.2013.11.021 
47. Kleberg JL, Riby PD, Fawcett C, Avdic HB, Frick MA, Brocki K, Högström J, Serlachius E, Nordgren A, Willfors C (2020) Williams syndrome: reduced attention to other's eyes in a hypersocial phenotype. PsyArXiv December 16, 2020. https://doi.org/10. 31234/osf.io/tp3yz.

48. Michelini G, Eley TC, Gregory AM, McAdams TA (2015) Aetiological overlap between anxiety and attention deficit hyperactivity symptom dimensions in adolescence. J Child Psychol Psychiatry 56(4):423-431. https://doi.org/10.1111/jcpp.12318

49. Hegerl U, Hensch T (2014) The vigilance regulation model of affective disorders and ADHD. Neurosci Biobehav Rev 44:45-57. https://doi.org/10.1016/j.neubiorev.2012.10.008

50. Sergeant J (2000) The cognitive-energetic model: an empirical approach to attention-deficit hyperactivity disorder. Neurosci Biobehav Rev 24(1):7-12. https://doi.org/10.1016/S01497634(99)00060-3

51. Strauß M, Ulke C, Paucke M, Huang J, Mauche N, Sander C, Stark T, Hegerl U (2018) Brain arousal regulation in adults with attention-deficit/hyperactivity disorder (ADHD). Psychiatry Res 261:102-108. https://doi.org/10.1016/j.psychres.2017.12.043

52. Doherty-Sneddon G, Riby DM, Calderwood L, Ainsworth L (2009) Stuck on You: Face-to-Face Arousal and Gaze Aversion in Williams Syndrome. Cogn Neuropsychiatry 14(6):510-523. https://doi.org/10.1080/13546800903043336

53. Hilt LM, Leitzke BT, Pollak SD (2017) Can't Take My Eyes off of You: Eye Tracking Reveals How Ruminating Young Adolescents Get Stuck. J Clin Child Adolesc Psychol 46(6):858-867. https:// doi.org/10.1080/15374416.2015.1121824
54. Faraone SV, Banaschewski T, Coghill D, Zheng Y, Biederman J, Bellgrove MA, Newcorn JH, Gignac M, Al Saud NM, Manor I, Rohde LA, Yang L, Cortese S, Almagor D, Stein MA, Albatti TH, Aljoudi HF, Alqahtani MMJ, Asherson P, Atwoli L, Bölte S, Buitelaar JK, Crunelle CL, Daley D, Dalsgaard S, Döepfner M, Espinet S, Fitzgerald M, Franke B, Haavik J, Hartman CA, Hartung CM, Hinshaw SP, Hoekstra PJ, Hollis C, Kollins SH, Sandra Kooij JJ, Kuntsi J, Larsson H, Li T, Liu J, Merzon E, Mattingly G, Mattos P, McCarthy S, Mikami AY, Molina BSG, Nigg JT, Purper-Ouakil D, Omigbodun OO, Polanczyk GV, Pollak Y, Poulton AS, Rajkumar RP, Reding A, Reif A, Rubia K, Rucklidge J, Romanos M, Ramos-Quiroga JA, Schellekens A, Scheres A, Schoeman R, Schweitzer JB, Shah H, Solanto MV, Sonuga-Barke E, Soutullo C, Steinhausen H-C, Swanson JM, Thapar A, Tripp G, van de Glind G, van Brink W, den; Van der Oord, S., Venter, A., Vitiello, B., Walitza, S., Wang, Y. (2021) The World Federation of ADHD International Consensus Statement: 208 Evidence-Based Conclusions about the Disorder. Neurosci Biobehav Rev. https:// doi.org/10.1016/j.neubiorev.2021.01.022

Publisher's Note Springer Nature remains neutral with regard to jurisdictional claims in published maps and institutional affiliations. 